\title{
Continuous Characterization of Permittivity over a Wide Bandwidth Using a Cavity Resonator
}

\author{
Rehab S. Hassan ${ }^{1}$ Sung Ik Park ${ }^{2} \cdot$ Ashwini Kumar Arya ${ }^{2} \cdot$ Sanghoek Kim,
}

\begin{abstract}
We examine a rectangular cavity resonator method to accurately characterize the complex permittivity of dielectric materials over a wide frequency range of $1-5 \mathrm{GHz}$ by exploiting the fundamental mode and higher-order $\mathrm{TE}_{(1,0, l)}$ modes. For this purpose, a rectangular waveguide is coupled with a cavity resonator through a large inductive aperture. The permittivity characterization at both even and odd $\mathrm{TE}_{(1,0, l)}$ modes enables continuous determination of the permittivity over operating frequencies. The characterization of the permittivity for even $\mathrm{TE}_{(1,0, l)}$ modes suffers from potential errors due to the displacement of materials. This paper also proposes a method to compensate for these errors and improve the accuracy in the even modes. The experimental results of the fabricated cavity are presented using different materials (frequency-independent and frequency-dependent). The measured complex permittivity results show a good agreement with the reported results over a wide bandwidth available in the literature.
\end{abstract}

Key Words: Aperture Size, Cavity Resonator, Complex Permittivity, Perturbation Technique, RF/Microwave Sensor.

\section{INTRODUCTION}

The microwave dielectric properties of materials have been widely used for understanding the behavior and the characteristics of various materials [1]. The difference in dielectric materials drives various losses and reflections for microwave frequencies. The measurement of the permittivity of materials has been demanded in many fields such as the food industry [2] and medicine [3].

Various methods have examined the complex permittivity of materials as in [4]. Among such methods, the rectangular cavity resonator is widely used to accurately detect the complex permittivity of low- and medium-loss materials [5-8]. However, the drawback of the conventional cavity resonator method is that the bandwidth of the measurement is quite narrow.
In an effort to enhance the bandwidth of the measurement, previous works characterized permittivity in multiple odd $\mathrm{TE}_{(1,0, l)}$ resonant modes [8]. The cavity method requires the material under test (MUT) to be placed where an E-field is strongest to maximize the perturbation [9] for all the modes (even and odd). In the even modes, the MUT position should be adjusted for each mode, so that it is located at the maximum E-field. A small displacement of the MUT from the maximum E-field position may introduce errors in the permittivity characterization. Conversely, in the odd modes, the maximum E-field always occurs at the center of the cavity. Therefore, the permittivity is usually in the odd modes only [5-8], with the position of the MUT fixed at the center of the cavity. As a result, the sampling of permittivity is sparse.

This study utilizes the multi-TE $(1,0, l)$ (both even and odd) modes for cavity perturbation to obtain complex permittivity

Manuscript received May 20, 2019 ; Revised August 2, 2019 ; Accepted October 21, 2019. (ID No. 20190520-038J)

${ }^{1}$ Department of Biomedical Engineering, Kyung Hee University, Yongin, Korea.

${ }^{2}$ Department of Electronics Engineering, Kyung Hee University, Yongin, Korea.

"Corresponding Author: Sanghoek Kim (e-mail: sanghoek@khu.ac.kr)

This is an Open-Access article distributed under the terms of the Creative Commons Attribution Non-Commercial License (http://creativecommons.org/licenses/by-nc/4.0) which permits unrestricted non-commercial use, distribution, and reproduction in any medium, provided the original work is properly cited.

(c) Copyright The Korean Institute of Electromagnetic Engineering and Science. All Rights Reserved. 
over broad bandwidth. By characterizing permittivity in the both even modes and odd modes, we increase the sampling density of the permittivity characterization within the frequency band. To suppress the displacement error in the even modes, this work proposes finding the maximum E-field spot directly from the $S_{11}$ response. A commercial electromagnetic simulation and measurement results show that the complex permittivities of all the MUTs are observed with high accuracy in the odd and even $\mathrm{TE}_{(1,0, l)}$ modes across the broadband from $1 \mathrm{GHz}$ to $5 \mathrm{GHz}$

\section{OVERALL SYSTEM STRUCTURE}

For the reflectivity study, an individual coaxial-to-waveguide transition is loaded into the cavity as illustrated in Fig. 1(a). A

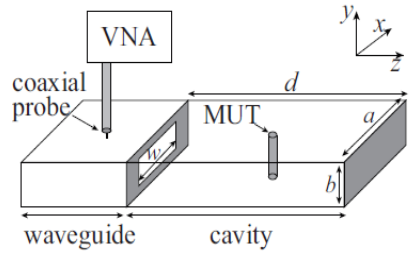

(a)

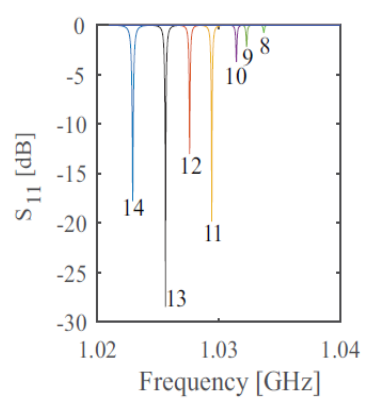

(b)
Fig. 1. (a) Schematic diagram of the structure. (b) Simulated reflection coefficient $S_{11}$ in the $\mathrm{TE}_{(1,0,1)}$ mode depending on the aperture width $w$, where the aperture height is $2 \mathrm{~cm}$. Numbers under the curves indicate the $w$ of each curve in centimeters. $w=13 \mathrm{~cm}$ shows critical coupling in the $\mathrm{TE}_{(1,0,1)}$ mode, and it is chosen as the aperture width.
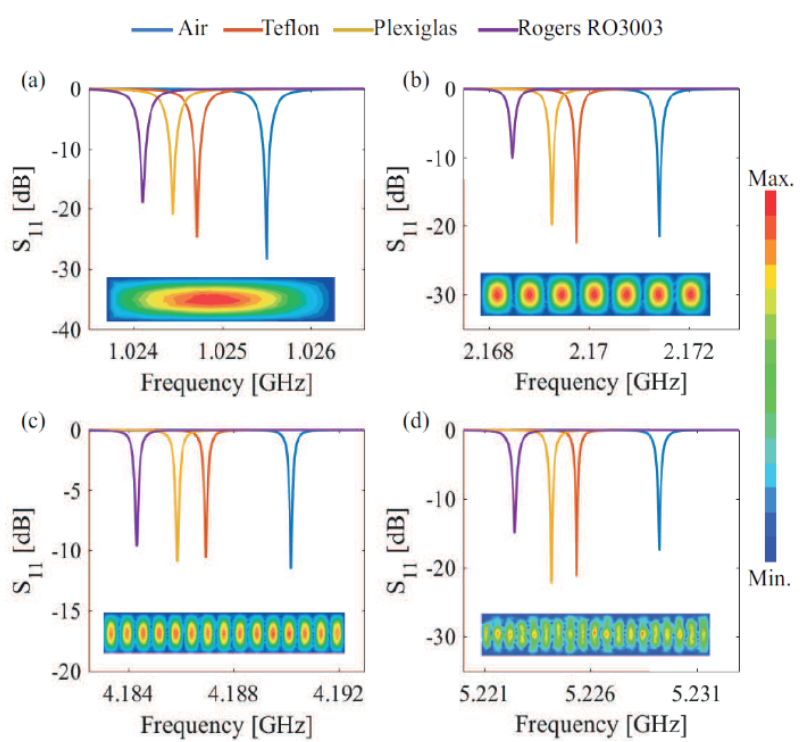

Fig. 2. Simulated $S_{11}$ the curves in the odd modes for different materials and the field distributions inside the cavity (inset). Resonant frequencies of the $\mathrm{TE}_{(1,0,1)}$ mode at $1 \mathrm{GHz}(\mathrm{a})$, $\mathrm{TE}_{(1,0,7)}$ mode at $2 \mathrm{GHz}(\mathrm{b}), \mathrm{TE}_{(1,0,15)}$ mode at $4 \mathrm{GHz}(\mathrm{c})$, and $\mathrm{TE}_{(1,0,19)}$ mode at $5 \mathrm{GHz}(\mathrm{d})$. closed-circuited metallic waveguide is integrated into the metallic cavity using an inductive aperture for the energy transaction. The system is designed to maximize power transfer to the cavity in the lowest mode by matching the impedance between the waveguide and the cavity with the essential aperture size in between [9] and [10]. As shown in Fig. 2(b), when the aperture height is fixed at $2 \mathrm{~cm}$ at $f_{(1,0,1)}$, the aperture width $w=13 \mathrm{~cm}$ has a minimum reflection coefficient $S_{11}$ of the cavity. The height $b$ and the width $w$ of the rectangular aperture are accordingly determined to be $2 \mathrm{~cm}$ and $13 \mathrm{~cm}$, respectively. The enclosed space inside the cavity is filled with air.

\section{CAVITY RESONATOR METHOD}

In this work, the cavity length $\mathrm{d}$ along the $z$-axis is designed to be longer than the other dimensions: $(d=55 \mathrm{~cm}) \gg>(a=15$ $\mathrm{cm}) \gg(b=3 \mathrm{~cm})$. This highly anisotropic design has a series of $\mathrm{TE}_{(1,0, l)}$ resonant modes in the frequency band of interest. As a result, the resonant frequency between $1 \mathrm{GHz}$ and $5 \mathrm{GHz}$ has a simple expression given in [5]:

$$
\mathrm{TE}_{(1,0, \mathrm{l})}=\frac{1}{2 \sqrt{\epsilon_{0} \mu_{0}}} \sqrt{\left(\frac{l}{a}\right)^{2}+\left(\frac{l}{d}\right)^{2}},
$$

where $\epsilon_{0}$ and $\mu_{0}$ are the permittivity and permeability of free space, respectively.

The cavity resonator method deduces the complex permittivity of the MUT by measuring how much the resonant frequency is shifted. Eqs. (2), and (3) are used to determine the complex permittivity $\epsilon_{r}$ of materials [8]:

$$
\begin{gathered}
\epsilon_{r}^{\prime}=1+\frac{2 \alpha V_{0}}{V_{s}}\left(\frac{f_{0}-f_{s}}{f_{s}}\right), \\
\epsilon_{r}^{\prime \prime}=\frac{\alpha V_{0}}{V_{s}}\left(\frac{1}{Q_{s}}-\frac{1}{Q_{0}}\right), \\
\alpha=\frac{V_{0}}{V_{s}}\left(\frac{\int_{V S} \boldsymbol{E} \cdot \boldsymbol{E}^{*} d V}{\int_{V 0} \boldsymbol{E}_{0} \cdot \boldsymbol{E}^{*} d V}\right),
\end{gathered}
$$

where $\epsilon_{r}^{\prime}$ and $\epsilon_{r}^{\prime \prime}$ are the real and imaginary part of complex permittivity, respectively, as $\epsilon_{r}=\epsilon_{r}^{\prime}-i \epsilon_{r}^{\prime \prime}$, and $V_{S}$ and $V_{0}$ are the volumes of the MUT and cavity, respectively. The MUT rod has a radius of $0.3 \mathrm{~cm}$ and height $b . Q_{0}$ and $Q_{s}$ are the $Q$ factors of the unperturbed and perturbed cavities, respectively. To maximize sensitivity, the MUT is placed where the E-field is strongest. In the odd modes, this location is always at the center of the cavity. The resonant frequency shifts with three different materials in the selected odd $\mathrm{TE}_{(1,0,1)}$ modes are demonstrated in Fig. 2.

To obtain a denser sampling of permittivity, the permittivity 
in the even modes should also be characterized. However, the location of the maximum E-field changes mode to mode in the even modes as shown in the inset of Fig. 3. Theoretically, the E-field of the $\mathrm{TE}_{(1,0,1)}$ mode has a maximum in every position of $z_{(\max , i)}=d(2 i-1) / 2 l$, where $i$ is an integer between 1 and $l$. Practically, the MUT position can be adjusted accordingly by having several holes at various locations or a rail way along the length of the resonant cavity. Nevertheless, any small deviation of the MUT position from the maximum E-field position in practice directly brings about errors in permittivity measurements at the even modes. A method to minimize such errors is described as follows.

The dielectric constant equation given by (2) is based on the fact that the MUT is placed where $|\mathrm{E}|$ is at maximum and that $|\mathrm{E}|$ distributes sinusoidally in the cavity. If the MUT is displaced from the maximum position by $\Delta z$, where $|\mathrm{E}| \approx$ $\left|\mathrm{E}_{\text {max }} \cos \frac{2 \pi \Delta}{\lambda}\right|$, the resonant frequency shift scales down according to the term $\left|\cos ^{2} \frac{2 \pi \Delta z}{\lambda}\right|$ as in the following equation [11]:

$$
\frac{f_{0}-f_{s}}{f_{0}} \approx \frac{\int_{V_{s}} \Delta \epsilon_{0}\left|E_{0}\right| \mathrm{dV}}{2 \int_{V_{0}} \epsilon_{0}\left|E_{0}\right| \mathrm{dV}} \approx \frac{V_{s} \Delta \epsilon_{0} \cos ^{2} \frac{2 \pi \Delta \mathrm{z}}{\lambda}}{\frac{V_{0} \epsilon_{0}}{2}}
$$

The above equation demonstrates that the frequency shift is

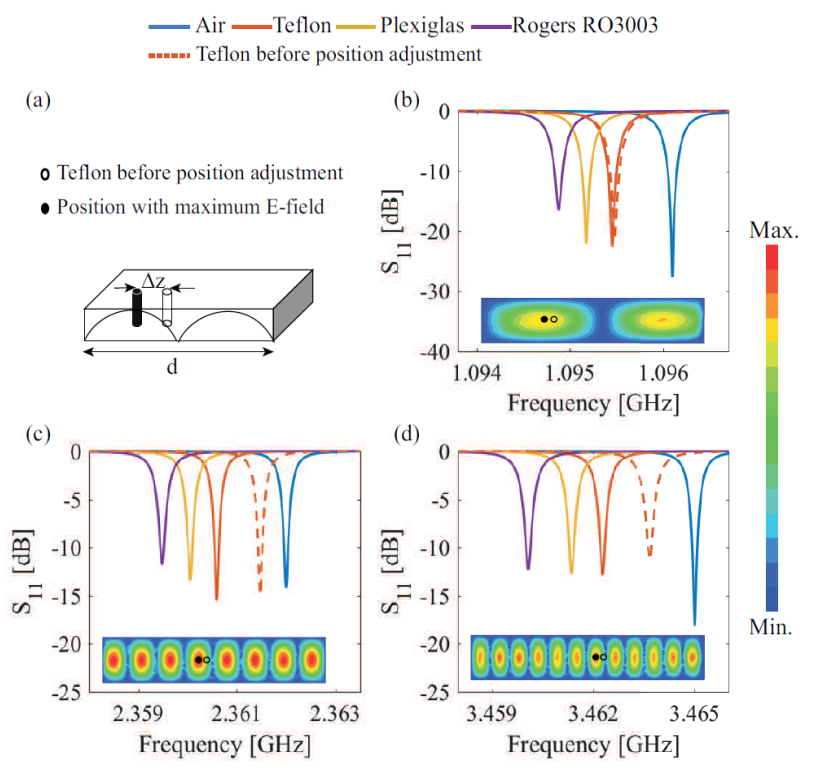

Fig. 3. (a) In the even modes, the positions of the maximum Efield can be found at the points where $S_{11}$ is maximally shifted. The shifted $S_{11}$ curves in the selected even modes due to various MUTs are shown. The dashed red curves show the $S_{11}$ curve with Teflon at arbitrary positions (empty dots). When Teflon is replaced at the position of the maximum E-field (filled dots), $S_{11}$ is maximally shifted (red curves). (b) The $\mathrm{TE}_{(1,0,2)}$ mode at $1 \mathrm{GHz}$, (c) $\mathrm{TE}_{(1,0,8)}$ mode at $2 \mathrm{GHz}$, and $(\mathrm{d}) \mathrm{TE}_{(1,0,12)}$ mode at 3 $\mathrm{GHz}$. at maximum when $\Delta z=0$. This reduces the expression of (5) back to (2) as expected. In other words, the position of the maximum E-field can be found by finding the position where the frequency shift is at maximum. By re-positioning the MUT to the spot of the maximum frequency shift, the error due to displacement is minimized. To illustrate the shifts of the resonant frequency for Teflon at $\mathrm{TE}_{(1,0,2)}, \mathrm{TE}_{(1,0,8)}$, and $\mathrm{TE}_{(1,0,12)}$ are shown in Fig. 3 for the even modes. When Teflon is misplaced from the position of the maximum E-field, the resonant frequency shifts are scaled down. Once the position of the MUT is decided upon to yield the maximum frequency shift with one material, the permittivity of other materials, such as Plexiglas and Rogers RO3003, can be measured sequentially in the same position, facilitating the characterization process of multiple MUTs.

\section{MEASUREMENT SETUP}

To validate the idea of the proposed cavity, the system is fabricated. The proposed system walls are made with aluminum material that has a conductivity of $\sigma_{a}\left(\sigma_{a}=3.816 \times 10^{7}\right)$. The walls are coated with a copper sheet to increase its conductivity as shown in the Fig. 4(a). The hole (rail way) is made in the proposed cavity starting from the center of the cavity to measure the complex permittivity of both modes (odd and even) as shown in Fig. 4(b). Fig. 4(c) illustrates the different MUTs used in the permittivity measurements of Plexiglas and fat phantom tissue. The unknown permittivity of MUTs can be calculated from Eqs. (2) and (3).

The fat phantom tissue, the permittivity of which is frequency dependent, is made as describe in [12]. Recently, various studies

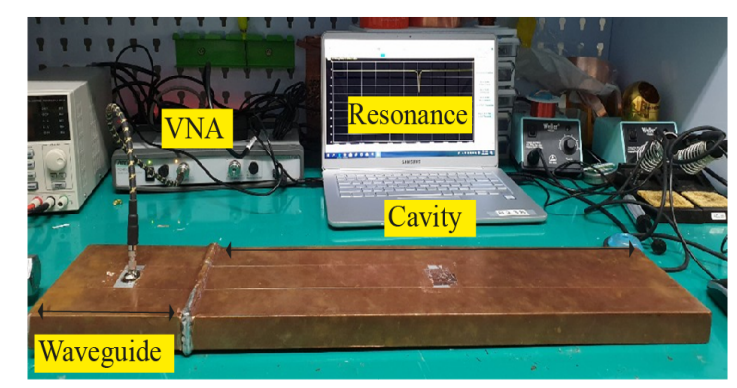

(a)

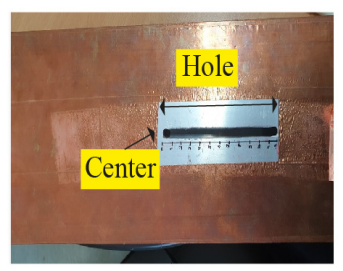

(b) (c)

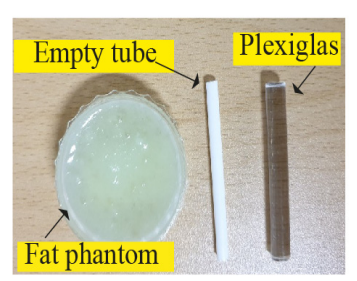

Fig. 4. (a) Setup of the measurements. (b) Hole size. (c) MUT samples. 
have observed fat thickness in the human body using a $\mathrm{RF} /$ microwave biosensor [13]. Phantom tissue has been used in many studies to observe the efficacy of the RF/microwave biosensor. Therefore, analyzing the permittivity of the fat phantom tissue, which should have a permittivity similar to that of the human fat, is essential. The fat phantom tissue is filled inside the empty tube to be inserted inside the proposed cavity to observe its permittivity. In the odd mode, the MUTs are placed at the center of the proposed cavity. However, in the even mode, the MUTs are inserted where the maximum shift occurs. The MUTs are moved through the hole of the rail as shown in Fig. 4(b). Then, the remaining open area of the hole (rail way) is closed by a copper sheet to reduce the air gap and prevent any leakage, and to reduce the measurement errors of the complex permittivity.

Prior to the measurements, an MS46122B vector network analyzer (VNA) is kept in calibration mode for one port. The entire setup is calibrated using three independent standards, namely open, short, and load, to reduce the effect of undesired losses and improves the accuracy of the measurement.

\section{RESULTS AND DiSCUSSION}

In the case of simulation, from the $S_{11}$ responses of the odd and even modes, $f_{(1,0,1)}$ to $f_{(1,0,19)}$, as in Figs. 2 and 3 for the MUTs, the resonant frequencies $f_{0}$ and $f_{s}$ are extracted to determine the real part of the permittivity. Then $Q_{0}$ and $Q_{s}$ are considered to determine the imaginary part of permittivity. The $Q$ factor is calculated from the 3-dB bandwidth. While $Q_{0}$ ranges from $\left(4.7697 \times 10^{3}-1.8061 \times 10^{4}\right)$, the $Q_{s}$ of Teflon, Plexiglas, and Rogers RO3003 are distributed as $\left(4.7048 \times 10^{3}-1.7160 \times 10^{4}\right),\left(4.6778 \times 10^{3}-1.6744 \times\right.$ $\left.10^{4}\right)$, and $\left(4.6340 \times 10^{3}-1.6150 \times 10^{4}\right)$, respectively.

Fig. 5 summarizes the real and imaginary parts of three perturbed MUTs obtained from a simulation over a series of $\mathrm{TE}_{(1,0, l)}$ modes. The complex permittivities of Teflon, Plexiglas,

l of TE $(1,0,1)$ modes

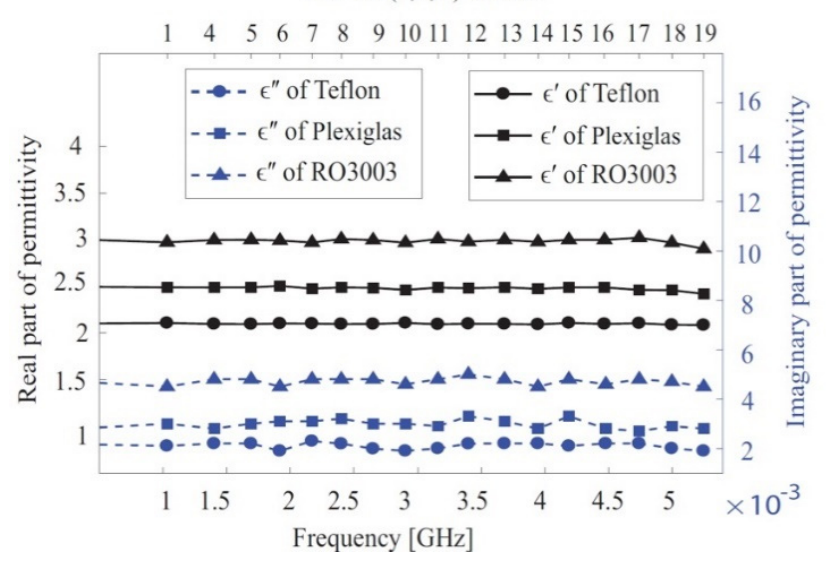

Fig. 5. Complex permittivity of MUTs.

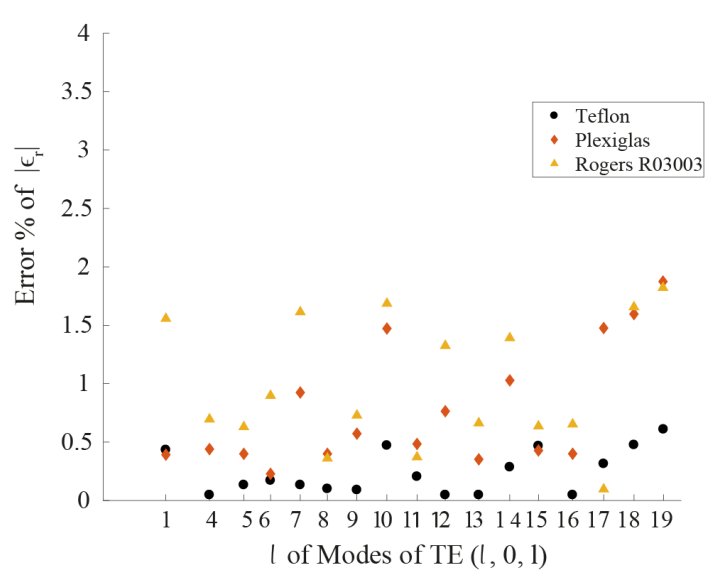

Fig. 6. Complex permittivity $\left|\epsilon_{r}\right|$ error of materials.

and Rogers RO3003 are originally set in the simulation as (2.1 $-0.0021 i, 2.5-0.003 i$, and $3.02-0.0048 i)$, respectively. The restored complex permittivities in Fig. 5 from the proposed method show $\left|\epsilon_{r}\right|$ errors less than $1 \%$ for Teflon and less than 2 $\%$ for Plexiglas and Rogers RO3003, as shown in Fig. 6. From the simulation, the mean values of the complex permittivities of Teflon, Plexiglas, and Rogers RO3003 at $1-5 \mathrm{GHz}$ are $(2.1000$ $\pm 0.0015-0.002 i \pm 0.5088),(2.4805 \pm 0.0045-0.0030 i \pm$ $0.6009)$, and $(2.9888 \pm 0.0064-0.0047 i \pm 0.7238)$, respectively.

In the case of measurements, we examine the complex permittivity of both odd and even modes at $f_{(1,0, l)}$. To prove the concept, we observe the odd modes at 1,3 , and $5 \mathrm{GHz}$ and the even modes at 2 and $4 \mathrm{GHz}$. In the first step, we study the complex permittivity of Plexiglas rod, with Plexiglas being frequency independent. The Plexiglas rod has a height of $\mathrm{b}$ and a radius of $0.3 \mathrm{~cm}$. Fig. 7 illustrates the complex permittivity of the Plexiglas rod with a comparison with [14].

Next, we study the complex permittivity for the fat phantom tissue. Fig. 8 shows that the permittivity measurement of the fat phantom tissue has the same trend as in the literature over a wide bandwidth $[12,15]$. The permittivity of the fat phantom tissue decreases by increasing the frequency. Therefore, the fat

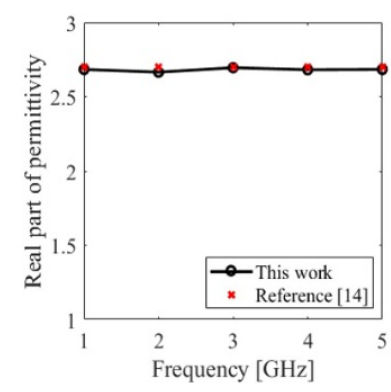

(a)

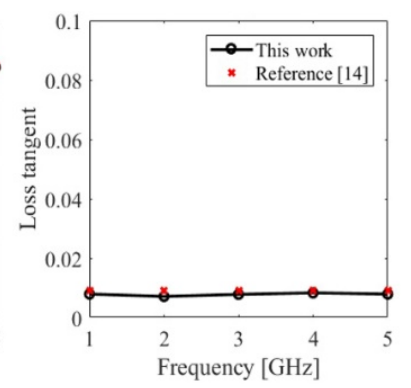

(b)
Fig. 7. The complex permittivity of Plexiglas. (a) The real part of the permittivity of Plexiglas. (b) The loss tangent of Plexiglas. 


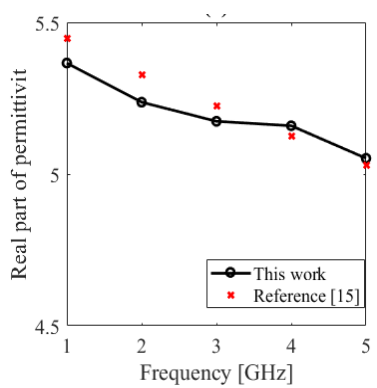

(a)

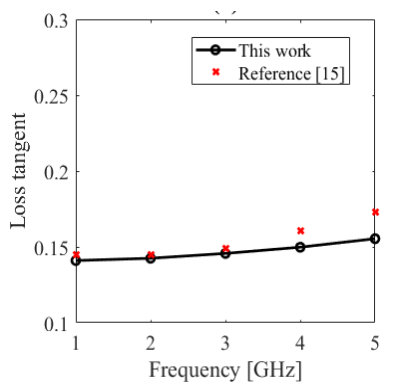

(b)
Fig. 8. Complex permittivity of fat phantom tissue. (a) Real part of the permittivity of the fat phantom tissue. (b) Loss tangent of the fat phantom tissue.

phantom tissue can be used instead of real fat tissue for experiments that require human/animal fat tissues.

\section{CONCLUSION}

The proposed rectangular cavity resonator is presented to characterize the complex permittivity of low-loss materials in the range of $1-5 \mathrm{GHz}$ for use in various applications. This method allows the accurate measurement of the permittivity of MUTs in a discrete, dense set of frequencies over a broad bandwidth.

This work was supported by the National Research Foundation of Korea (No. NRF-2017 R1C1B2009892 and NRF2018R1A6A1A03025708).

\section{REFERENCES}

[1] L. F. Chen, C. K. Ong, C. P. Neo, V. V. Varadan, and V. K. Varadan, Microwave Electronics: Measurement and Materials Characterization. Chichester, UK: John Wiley \& Sons, 2004.

[2] M. S. Venkatesh and G. S. V. Raghavan, "An overview of dielectric properties measuring techniques," Canadian Biosystems Engineering, vol. 47, no. 7, pp. 15-30, 2005.

[3] A. Martellosio, M. Pasian, M. Bozzi, L. Perregrini, A. Mazzanti, F. Svelto, P. E. Summers, G. Renne, L. Preda, and M. Bellomi, "Dielectric properties characterization from 0.5 to $50 \mathrm{GHz}$ of breast cancer tissues," IEEE Transactions on Microwave Theory and Techniques, vol. 65, no. 3, pp. 998-1011, 2016.

[4] R. N. Clarke, A. P. Gregory, D. Cannell, M. Patrick, S. Wylie,
I. Youngs, and G. Hill, $A$ Guide to the Characterisation of Dielectric Materials at RF and Microwave Frequencies. London: The Institute of Measurement and Control, 2003.

[5] K. Saeed, R. D. Pollard, and I. C. Hunter, "Substrate integrated waveguide cavity resonators for complex permittivity characterization of materials," IEEE Transactions on Microwave Theory and Techniques, vol. 56, no. 10, pp. 2340-2347, 2008.

[6] A. K. Jha and M.J. Akhtar, "A generalized rectangular cavity approach for determination of complex permittivity of materials," IEEE Transactions on Instrumentation and Measurement, vol. 63, no. 11, pp. 2632-2641, 2014.

[7] N. D. Orloff, J. Obrzut, C. J. Long, T. Lam, P. Kabos, D. R. Novotny, J. C. Booth, and J. A. Liddle, "Dielectric characterization by microwave cavity perturbation corrected for nonuniform fields," IEEE Transactions on Microwave Theory and Techniques, vol. 62, no. 9, pp. 2149-2159, 2014.

[8] A. Kik, "Complex permittivity measurement using a ridged waveguide cavity and the perturbation method," IEEE Transactions on Microwave Theory and Techniques, vol. 64, no. 11, pp. 3878-3886, 2016.

[9] D. M. Pozar, Microwave Engineering, 3rd ed. Hoboken, NJ: John Wiley \& Sons, 2005.

[10] G. L. Matthaei, L. Young, and E. M. Jones, Microwave Filters, Impedance-Matching Networks, and Coupling Structures. New York, NY: McGraw-Hill, 1964.

[11] R. F. Harrington, Time-Harmonic Electromagnetic Fields. New York, NY: McGraw-Hill, 1961.

[12] T. Yilmaz, R. Foster, and Y. Hao, "Broadband tissue mimicking phantoms and a patch resonator for evaluating noninvasive monitoring of blood glucose levels," IEEE Transactions on Antennas and Propagation, vol. 62, no. 6, pp. 3064-3075, 2014.

[13] J. Park, J. Lee, B. Lee, A. S. Y. Poon, S. Lee, and S. Kim, "Measuring abdominal fatness using principle of Salisbury screen," Electronics Letters, vol. 53, no. 14, pp. 908-910, 2017.

[14] M. Shete, M. Shaji, and M. J. Akhtar, "Design of a coplanar sensor for RF characterization of thin dielectric samples," IEEE Sensors Journal, vol. 13, no. 12, pp. 4706-4715, 2013.

[15] S. Gabriel, R. W. Lau, and C. Gabriel, "The dielectric properties of biological tissues: III. Parametric models for the dielectric spectrum of tissues," Physics in Medicine E Biology, vol. 41, no. 11, pp. 2271-2293, 1996. 


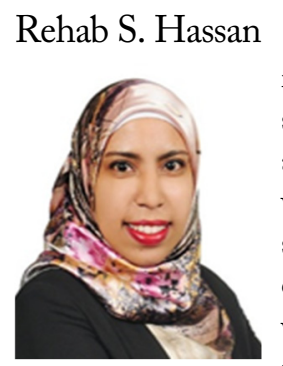

received her B.S. in biomedical engineering and systems from El-Shorouk Academy, Egypt, in 2010 and her M.S. in biomedical engineering and systems with specialization in photonics from Cairo University, Egypt, in 2013. She served as a senior biomedical engineer in Promega Corporation Medical Devices with Delta Trading and Development Company, Ltd., Egypt, from 2011 to 2016. She is currently pursuing her $\mathrm{Ph} . \mathrm{D}$. degree in biomedical engineering at Kyung Hee University, South Korea. Her research interests include the application of $\mathrm{RF} /$ microwave technology in medicine, electromagnetic theories, passive implantable sensor, and wireless power transmission.
Sung Ik Park

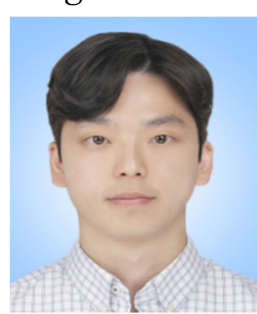

received his B.S. degree in electrical engineering from Kyung Hee University, Yongin, Korea, in 2019. He is currently working toward his M.S. in electrical engineering from Kyung Hee University. His research interests include microwave antennas, radio frequency cavity resonator, and wireless power transmission.

\section{Ashwini Kumar Arya}

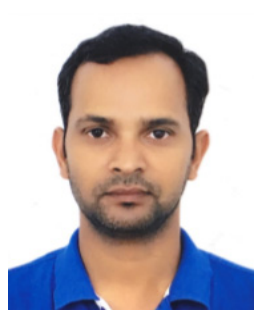

received his B.E. in electronics and communication engineering in 2005 from HNB Garhwal University, India and his M.Tech. in electronics communication engineering in 2007 from GBPUAT Pantnagar, India. He received his $\mathrm{Ph} . \mathrm{D}$. in radio frequency and microwave engineering in 2013 from the Indian Institute of Technology, India. He worked as a postdoctoral researcher from 2014 to 2016 in the Department of Electrical Engineering, KAIST, Daejeon, South Korea. Before joining Kyung Hee University, he worked as an assistant professor in the Department of Electronics and Communication Engineering, GBPUAT, India. Currently he works as a research professor at the Institute for Wearable Convergence Electronics, Kyung Hee University, Yongin, South Korea, and is also affiliated with the Department of Electronics Engineering, Kyung Hee University, Yongin, South Korea. His research interests include the applications of radio frequency engineering, electromagnetic theory in wireless communication and antenna design and technology for various applications.

\section{Sanghoek Kim}

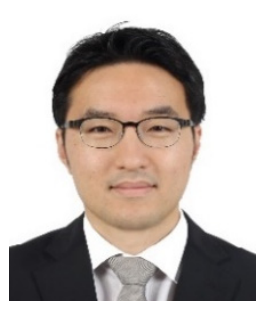

received his B.S. degree with a double major in electrical engineering and mathematical science from Seoul National University, Korea, in 2007, and his M.S. and Ph.D. degrees in electrical engineering from Stanford University, USA, in 2013. He was a recipient of the Kwanjeong Scholarship during his study. After graduation, he worked at Qualcomm Inc., as a signal/power integrity engineer and in $\mathrm{Si}^{-}$ Beam, Inc., as an mm-wave system engineer. In 2016, he joined the Department of Electronics Engineering, Kyung Hee University as an assistant professor. Currently, his research interests include the applications of radio frequency technology and electromagnetics theory in wireless interface with bio-implantable devices, bio-medicine, and radar technologies. 